\title{
Evaluation of meisoindigo, an indirubin derivative: in vitro antileukemic activity and in vivo pharmacokinetics
}

\author{
MENG HUANG ${ }^{1,2}$, HAI-SHU LIN ${ }^{1}$, YING SHIUAN LEE ${ }^{1,3}$ and PAUL C. HO ${ }^{1}$ \\ ${ }^{1}$ Department of Pharmacy, Faculty of Science, National University of Singapore, Singapore 117543, Republic of Singapore; \\ ${ }^{2}$ Department of Pharmacology, Perelman School of Medicine, University of Pennsylvania, Philadelphia, PA 19104, USA; \\ ${ }^{3}$ Department of Pharmacy, University of Gothenburg, SE-405 30 Gothenburg, Sweden
}

Received April 21, 2014; Accepted June 6, 2014

DOI: $10.3892 /$ ijo.2014.2548

\begin{abstract}
Meisoindigo has been a routine therapeutic agent in the clinical treatment of chronic myelogenous leukemia (CML) in China since the 1980s. In the present study, the in vitro antileukemic activity of meisoindigo was investigated in acute promyelocytic leukemia (APL) cells, acute myeloid leukemia (AML) cells, and myelomonocytic leukemia cells (NB4, NB4.007/6, HL-60 and U937) comprising both retinoic acid-sensitive and retinoic acid-resistant cells. We found that meisoindigo effectively inhibited the growth and/or proliferation of these four cell types at $\mu \mathrm{M}$ levels. The effects of meisoindigo in these cells are related to its proliferation inhibition and apoptosis induction, and are independent of cell cycle arrest, indicating that meisoindigo could be possible in the treatment of APL, AML and retinoic acid resistant APL. The in vivo pharmacokinetics of meisoindigo and its major circulatory metabolites in rat plasma were then investigated by a newly developed and validated liquid chromatography-tandem mass spectrometry (LC-MS/MS) method. The profiles of plasma concentration versus time were plotted and the relevant pharmacokinetic parameters were calculated for meisoindigo and its reductive metabolites. The plasma concentrations of meisoindigo after oral administration were much lower than the in vitro $\mathrm{IC}_{50} \mathrm{~s}$ determined in the leukemic cells. The contradicting poor pharmacokinetic characteristics and the established clinical efficacy of meisoindigo could indicate the presence of active metabolites in vivo.
\end{abstract}

Correspondence to: Professor Paul C. Ho, Department of Pharmacy, Faculty of Science, National University of Singapore, 18 Science Drive 4, Singapore 117543, Republic of Singapore

E-mail: phahocl@nus.edu.sg

Abbreviations: AML, acute myeloid leukemia; APL, acute promyelocytic leukemia; CML, chronic myelogenous leukemia; EPI, enhanced product ion; ESI, electrospray ionization; LC-MS/MS, liquid chromatography-tandem mass spectrometry; MRM, multiple reaction monitoring; QTRAP, hybrid triple quadrupole linear ion trap

Key words: meisoindigo, acute promyelocytic leukemia, acute myeloid leukemia, reductive metabolites, pharmacokinetics, liquid chromatography-tandem mass spectrometry

\section{Introduction}

Meisoindigo, a second-generation derivative of indirubin, has been a routine therapeutic agent in the clinical treatment of CML in China since 1980s $(1,2)$. In the phase III clinical trial of meisoindigo involving 402 patients, it was shown that meisoindigo was equally efficient for both newly diagnosed and previously treated CML patients after oral administration. The hematological complete response (CR) and partial response (PR) rates, respectively, were 45.0 and $39.3 \%$ for newly diagnosed patients and 35.9 and $41.4 \%$ for previously treated patients (2). Meisoindigo was generally well tolerated. The most frequent side-effects were bone, joint and/or muscle pain of varying degrees when the dosage was more than the suitable one (1).

The molecular mechanism of action of meisoindigo is still not well understood. It appears that growth inhibition and apoptosis of the treated cancer cells might be the major mechanism of action of meisoindigo (2). Previous studies have indicated that meisoindigo strongly inhibits DNA biosynthesis in cancer cells and inhibits the assembly of microtubules $(3,4)$. Experimental results on the mouse leukemia L1210 cell cycle showed that meisoindigo induced accumulation of $\mathrm{S}$ phase cells. The movement of cells in G2+M phase to G1 phase may also be blocked to some extent (3). The induction of cancer cell differentiation associated with decreased $c$ - $m y b$ oncogene expression might also account for the anticancer action and low toxicity of meisoindigo (4). Another study indicated that the anti-angiogenesis effect of meisoindigo may contribute to the antileukemic effect of this drug (5).

As meisoindigo is clinically effective in the management of CML with high efficacy and low toxicity, it could be effective in the treatment of other types of myeloid leukemia including acute promyelocytic leukemia (APL), acute myeloid leukemia (AML) and myelomonocytic leukemia. However, the information on the effects of meisoindigo in these myeloid leukemias is still limited. One previous study reported that meisoindigo showed promising in vitro and in vivo activity against AML (6). In the present study, we tested the antileukemic effects of meisoindigo in the human NB4 (APL cells, FAB-M3), NB4.007/6 (retinoic acid-resistant cells derived from NB4), HL60 (AML cells, FAB-M2), and U937 (myelomonocytic leukemia cells, FAB-M5). Among them, NB4 and 
HL60 are retinoic acid-sensitive cells $(7,8)$, whereas NB4.007/6 and $\mathrm{U} 937$ are retinoic acid-resistant cells $(9,10)$.

To improve the understanding of its efficacy and safety characteristics, detailed investigation of meisoindigo metabolism is warranted. In our previous studies, the in vitro metabolic profiles of meisoindigo in rat, pig and human liver microsomes were explored from both the qualitative and quantitative aspects $(11,12)$. The in vivo metabolic profiles of meisoindigo in rat plasma, urine and feces were examined from the qualitative aspect (13). The major circulatory metabolites of meisoindigo in rat plasma were identified as 3,3 double bond reduction products by LC-MS/MS. However, to our knowledge, quantitative information relevant to the in vivo metabolic profile of meisoindigo is lacking. Another objective of this study was to elucidate the pharmacokinetic properties of meisoindigo and its major circulatory metabolites in rats following oral administration. The latter experiments served as an in vivo model to illustrate the metabolism and disposition of meisoindigo and its metabolites after oral administration.

\section{Materials and methods}

Chemicals and reagents. Cell culture medium and reagents, propidium iodide (PI), DMSO (molecular biology grade), sodium carboxymethyl cellulose (CMC-Na) and formic acid were purchased from Sigma Chemical Co. (St. Louis, MO, USA). Fetal bovine serum (FBS) was obtained from HyClone (Logan, UT, USA). RNase was bought from Roche (Basel, Switzerland). Meisoindigo was provided by the Institute of Materia Medica, Chinese Academy of Medical Sciences and Peking Union Medical College (Beijing, China). Indirubin (internal standard) was purchased from the National Institute for the Control of Pharmaceutical and Biological Products (Beijing, China). HPLC grade ethyl acetate, methanol and acetonitrile were purchased from Fisher Scientific Co. (Fair Lawn, NY, USA). Milli-Q water was obtained from a Millipore water purification system (Billerica, MA, USA) and used to prepare buffer solutions and other aqueous solutions.

Cell cultures. NB4 (APL cells, M3 subtype according to FAB) (7), NB4.007/6 (retinoic acid resistant cells developed through continuously culturing NB4 in the medium containing retinoic acid (9), HL60 (AML cells, M2 subtype according to FAB) (14), and U937 (myelomonocytic leukemia cells, M5 subtype according to FAB) $(10,15)$, were maintained in RPMI-1640 medium supplemented with $10 \%$ heat-inactive fetal bovine serum, $100 \mathrm{U} / \mathrm{ml}$ penicillin, and $100 \mu \mathrm{g} / \mathrm{ml}$ streptomycin in a humidified $5 \% \mathrm{CO}_{2}$ atmosphere at $37^{\circ} \mathrm{C}$. The cells were subjected to sub-culture by 1:2 dilution with fresh medium every 3 days. Cultured cells with a passage number of 10-20 were used in the experiments to reduce variability due to cell culture conditions.

Inhibitory effects of meisoindigo on the four human leukemic cells. The inhibitory effects of meisoindigo on the four human leukemic cells were tested by treating the cells with meisoindigo at various concentrations $(0,1,2,4,8,12,16,20 \mu \mathrm{M})$. The cells were cultured in 24 -well plates. The number of the cells was counted by trypan blue exclusion method. The initial cell numbers (NB4, NB4.007/6, HL60 and U937) were
$3 \times 10^{6}$ cell $/ \mathrm{ml}$. After incubation for 24,48 and $72 \mathrm{~h}$, the plates were taken out and the number of viable cells was counted. All experiments were carried out in eight replicates. The cell activity was defined as the number of viable cells in the test culture over the mean number of viable cells in the control. The $50 \%$ inhibitory concentration $\left(\mathrm{IC}_{50}\right)$ on cell activity was estimated based on the cell number obtained after incubation for $72 \mathrm{~h}$. The data were fitted to the Inhibitory Effect Sigmoid $\mathrm{E}_{\max }$ Model with the software, WinNonlin version 1.0 (Lexington, KY, USA). All regressions were carried out with equal weighting.

Flow cytometry analysis for apoptosis and cell cycle distribution. Apoptosis and cell cycle distribution of the tested leukemic cells were examined by flow cytometry analysis. The leukemic cells were treated with meisoindigo of various concentrations $(0,4,8,12,16$ and $20 \mu \mathrm{M})$ for 24,48 and $72 \mathrm{~h}$ in the respective 6-well plates. At the end of incubation, the cells were harvested, washed twice with $3 \mathrm{ml}$ PBS (pH 7.4 contain 1\% FBS), and then fixed with $70 \%$ ice-cold ethanol and kept at $-20^{\circ} \mathrm{C}$ for at least $24 \mathrm{~h}$. On the day before flow cytometry assay, the fixed leukemic cells were spun down, washed with $3 \mathrm{ml}$ PBS with $1 \%$ FBS and spun down again. The cells were then dyed in $500 \mu \mathrm{l}$ PBS containing $100 \mu \mathrm{g} / \mathrm{ml}$ propidium iodide (PI) and $100 \mu \mathrm{g} / \mathrm{ml} \mathrm{RNase}$ and stored at $4^{\circ} \mathrm{C}$ overnight. On the following day, the samples were first filtered through a $30 \mu \mathrm{m}$ pore size nylon mesh prior to the flow cytometry analysis. The samples were analyzed on a Coulter Epics Elite ESP flow cytometer (Beckman Coulter, FL, USA) equipped with a $15 \mathrm{~mW}$ argonion laser source of $488 \mathrm{~nm}$. Red fluorescence of propidium iodide (PI) was collected with a $610 \mathrm{~nm}$ bandpass filter. Typical flow rates were about 200 to 300 particles per sec. A total of 10,000 cells were analyzed per sample. The experiment was carried in triplicate. The data obtained were analyzed using WinMDI 2.8 software (La Jolla, CA, USA). From the DNA histogram, the percentages of cells in different cell cycle phases were determined. Cells with DNA concentration less than the G1 phase were regarded as apoptotic cells.

Statistics. Statistical analysis was performed by using SPSS 10.0 (Chicago, IL, USA) or GraphPad Prism 2.00 (La Jolla, CA, USA). All experimental data were analyzed with One-Sample Kolmogorov-Smirnov Test for their distribution. As the parameters were found to be normally distributed, data were expressed as mean \pm standard derivation (SD). $\mathrm{EC}_{50}$ on growth inhibition was compared at their $95 \%$ confidential level. The percentages of apoptotic cells at different meisoindigo concentrations were compared with the one-way ANOVA and the posthoc Tukey's test. A value of $p<0.05$ was adopted to indicate statistical significance.

Synthesis and purification of meisoindigo reductive metabolites. Synthetic routes of reductive metabolite standards (M1+M2 and M3+M4) are shown in Fig. 1 as previously described (11). The starting material of meisoindigo (50 mg) was dissolved in methanol $(100 \mathrm{ml})$. After adding $10 \%$ palladium on carbon $(10 \mathrm{mg})$, the mixture was placed inside the hydrogenator apparatus and kept shaking at 40 psi for $2 \mathrm{~h}$ at room temperature. The reaction mixture was filtered through filter paper and the filter pad was washed with distilled water. 


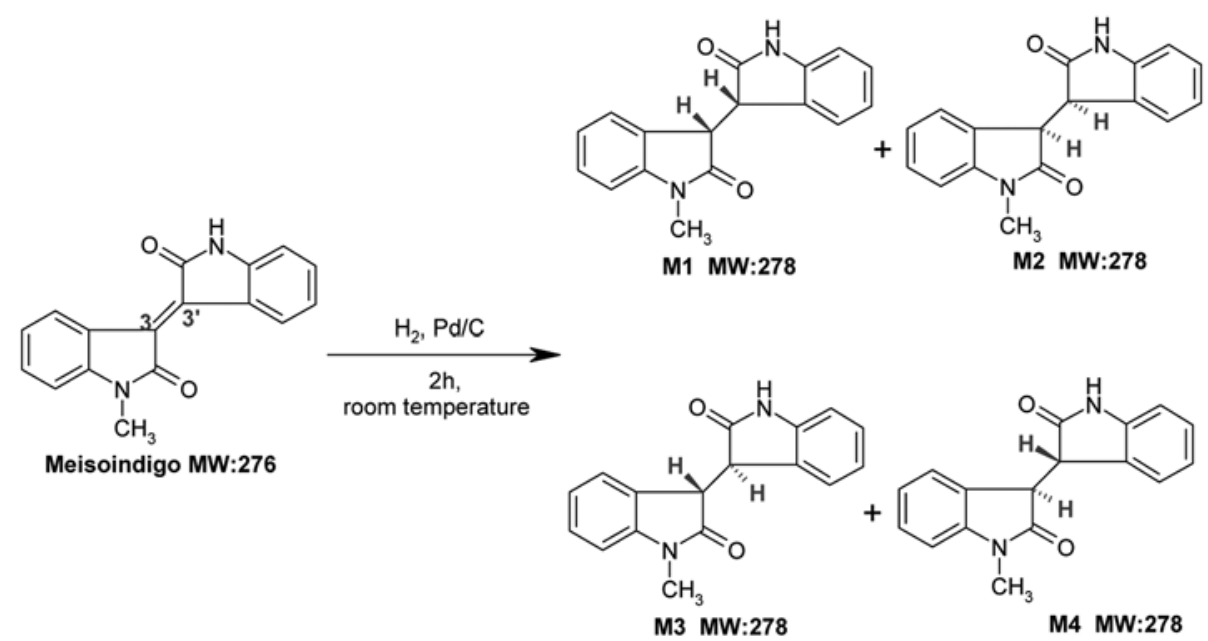

Figure 1. Synthetic routes of reductive metabolites of meisoindigo.

The filtrate was evaporated under reduced pressure and the precipitate was purified by Agilent 1100 preparative HPLC system (Palo Alto, CA, USA) on a Peeke Scientific Combi-A C18 column (50 x 20 mm, $5 \mu \mathrm{m}$ ) (Redwood City, CA, USA) with a guard cartridge at ambient temperature. The isocratic mobile phase consisted of 50\% water (solvent A) and 50\% methanol (solvent B) and was delivered at a flow rate of $4 \mathrm{ml} / \mathrm{min}$ within $20 \mathrm{~min}$. The UV absorbance was monitored at $254 \mathrm{~nm}$. The combined metabolites fractions (total M1+M2 and M3+M4) were evaporated under reduced pressure and stored at $-20^{\circ} \mathrm{C}$.

LC-MS/MS conditions for simultaneous quantification of meisoindigo and its reductive metabolites. The LC-MS/MS system consisted of an Agilent 1200 HPLC (Palo Alto, CA, USA) with a Q TRAP ${ }^{\text {TM }} 3200$ hybrid triple quadrupole linear ion trap mass spectrometer from Applied Biosystems/MDS Sciex (Concord, Ontario, Canada). Chromatographic separations for rat plasma samples were performed on a Phenomenex Luna C18 column (150 mm x $2.00 \mathrm{~mm}$ i.d., $5 \mu \mathrm{m}$ ) (Torrance, CA, USA) with a guard cartridge. The injection volume and column temperature were $5 \mu 1$ and $25^{\circ} \mathrm{C}$, respectively. The mobile phases consisted of $0.1 \%$ formic acid in water (solvent A) and acetonitrile (solvent B). The gradient elution was set at a flow rate of $0.3 \mathrm{ml} / \mathrm{min}$. The optimized linear gradient elution conditions were: 30 to $95 \%$ B over $13 \mathrm{~min}$, followed by an isocratic hold at $95 \% \mathrm{~B}$ for another $2 \mathrm{~min}$. At $15 \mathrm{~min}, \mathrm{~B}$ was returned to $30 \%$ in $1 \mathrm{~min}$ and the column was equilibrated for $14 \mathrm{~min}$ before the next injection. The total run time was $30 \mathrm{~min}$. During LC-MS/MS analysis, up to $4 \mathrm{~min}$ of the initial flow was diverted away from the mass spectrometer before the data acquisition. The mass spectrometer was operated in the positive ion mode with a TurboIonSpray source. Enhanced product ion (EPI) scans were used to investigate the fragmentation patterns of authentic standard compounds. A list of three Multiple Reaction Monitoring (MRM) transitions in Table I was selected for the quantification of meisoindigo, its reductive metabolites and indirubin (internal standard). The Q1, Q3, declustering potential (DP), and collision energy (CE) values in Table I were based on the MS/MS results of those respective standards. The other ionization parameters were as follows: curtain gas (CUR), 20 (arbitrary units); ion
Table I. MRM transition parameters for meisoindigo, its reductive metabolites and indirubin (internal standard) in rat plasma.

\begin{tabular}{lcccc}
\hline & $\begin{array}{c}\mathrm{Q} 1 \\
(\mathrm{amu})\end{array}$ & $\begin{array}{c}\mathrm{Q} 3 \\
(\mathrm{amu})\end{array}$ & $\begin{array}{c}\mathrm{DP} \\
(\mathrm{V})\end{array}$ & $\begin{array}{c}\mathrm{CE} \\
(\mathrm{eV})\end{array}$ \\
\hline Meisoindigo & 277.1 & 234.2 & 66 & 45 \\
Reductive metabolites & 279.1 & 147.1 & 61 & 30 \\
Indirubin (internal standard) & 263.1 & 190.1 & 80 & 50 \\
\hline
\end{tabular}

source gas 1 (GS1), 40 (arbitrary units); ion source gas 2 (GS2), 50 (arbitrary units); source temperature (TEM), $550^{\circ} \mathrm{C}$; entrance potential (EP), $10 \mathrm{~V}$. The dwell time of each MRM transition was $150 \mathrm{msec}$. The HPLC system and the mass spectrometer were controlled by Analyst ${ }^{\mathrm{TM}}$ 1.4.2 software from Applied Biosystems/MDS Sciex.

Preparation of standards and quality control samples. Stock solutions of meisoindigo and its reductive metabolites (M1+M2 and M3+M4) were prepared by dissolving the accurately weighed compounds in methanol to give a final concentration of $1 \mathrm{mg} / \mathrm{ml}$ for both. Solution of indirubin (internal standard) was prepared in methanol at the concentration $1 \mathrm{mg} / \mathrm{ml}$ and diluted to $1 \mu \mathrm{g} / \mathrm{ml}$ with methanol. Blank rat plasma (drug free) was obtained by centrifugation of predose rat blood. Calibration curves were prepared by spiking appropriate standard solutions of the parent drug meisoindigo and its reductive metabolites, respectively, to the blank plasma. Concentrations in plasma samples were $1,2.5,5,10,50,250$ and $500 \mathrm{ng} / \mathrm{ml}$ for meisoindigo and 2.5, 5, 50, 100, 250, 500 and 1,000 ng/ml for its reductive metabolites. Quality control (QC) samples were separately prepared in blank plasma samples at the concentration of 5,50 and $500 \mathrm{ng} / \mathrm{ml}$ for meisoindigo and its reductive metabolites, respectively.

Rat plasma sample preparation. Each rat plasma sample (45 $\mu \mathrm{l}$ ) was added with $5 \mu \mathrm{l}$ of internal standard indirubin $(100 \mathrm{ng} / \mathrm{ml})$. The samples were briefly mixed before extraction with 2 x 2-fold volume of cold ethyl acetate saturated with 
$\mathrm{H}_{2} \mathrm{O}$. The organic phases from the extracted plasma were combined and dried at $35^{\circ} \mathrm{C}$ under a gentle stream of nitrogen. The residue was reconstituted with $50 \mu$ l acetonitrile and $\mathrm{H}_{2} \mathrm{O}$ (1:1) for LC-MS/MS analysis.

\section{LC-MS/MS method validation}

Selectivity. Blank rat plasma were analyzed for interference using the proposed sample preparation procedure and LC-MS/MS conditions, compared to blank plasma spiking with meisoindigo and its reductive metabolites.

Calibration curve. Through the internal standard method, seven non-zero samples with meisoindigo and its reductive metabolites were used in establishing two standard calibration curves that covered the expected range. Calibration curves were generated using linear least square regression. The lower limit of quantification (LLOQ) of the assay was also determined.

Accuracy and precision. Intra-day accuracy and precision were determined by the analysis at the concentrations of $1,2.5$, $5,10,50,250$ and $500 \mathrm{ng} / \mathrm{ml}$ for meisoindigo and 2.5, 5, 50, 100, 250,500 and $1,000 \mathrm{ng} / \mathrm{ml}$ for its reductive metabolites. Three replicates of each concentration were analyzed within the same day for this purpose. Inter-day accuracy and precision were evaluated by the analysis carried out on three consecutive days. Accuracy was calculated as the percentage ratio of the mean of the measured concentration to the spiked concentration. Precision was expressed by the relative standard deviation.

Extraction recovery. The extraction recoveries of meisoindigo and its reductive metabolites at three quality control levels $(5,50$ and $500 \mathrm{ng} / \mathrm{ml})$ were evaluated in triplicate by comparing peak area ratios of meisoindigo and its reductive metabolites obtained from plasma samples with those obtained from the standard solutions at the same concentration.

Stability. For short-term stability, three aliquots of each of the low $(5 \mathrm{ng} / \mathrm{ml})$ and high $(500 \mathrm{ng} / \mathrm{ml})$ concentrations samples were prepared from the stock solutions and stored at $-80^{\circ} \mathrm{C}$. They were thawed at room temperature and kept at this temperature for $4 \mathrm{~h}$ on the same day before analyzing. For longterm stability, three aliquots of each of the 5 and $500 \mathrm{ng} / \mathrm{ml}$ samples were stored at $-80^{\circ} \mathrm{C}$ for 4 weeks before being thawed and analyzed. For freeze and thaw stability, three aliquots of 5 and $500 \mathrm{ng} / \mathrm{ml}$ samples underwent three freeze and thaw cycles for three consecutive days before being analyzed. For stock solution stability, three aliquots of the 5 and $500 \mathrm{ng} / \mathrm{ml}$ stock solutions were thawed and kept at room temperature for $6 \mathrm{~h}$ before evaluating. For post-preparative stability, three aliquots of 5 and $500 \mathrm{ng} / \mathrm{ml}$ samples were kept in the LC-MS/MS autosampler chamber for $8 \mathrm{~h}$ before being analyzed. Stability was expressed as the percentage ratio of the mean of the measured concentration to the spiked concentration.

Pharmacokinetic studies of meisoindigo and its reductive metabolites. The pharmacokinetic profiles of meisoindigo and its reductive metabolites after oral administration were studied in rats. The animal experimental protocols were reviewed and approved by the Institutional Animal Care and Use Committee of the National University of Singapore (NUS). For the study, four healthy male Sprague-Dawley (SD) rats (7-8 weeks of age, average $250 \mathrm{~g}$ ) were purchased from Animal Holding Unit, National University of Singapore. They were provided with a standard diet and water ad libitum. The room was kept on a 12/12-h light/dark cycle at a temperature of $23 \pm 1^{\circ} \mathrm{C}$ and relative humidity of $50 \pm 10 \%$. At least one week of acclimatization period was allowed for the rats prior to drug administration. The rats were fasted $12 \mathrm{~h}$ prior to administration of the dose and were fed $12 \mathrm{~h}$ after the dose. The dose was formulated in a mixture of meisoindigo suspended in $1 \%$ sodium carboxymethyl cellulose (CMC-Na) solution at a target concentration of $3.75 \mathrm{mg} / \mathrm{ml}$. The rats received meisoindigo solution by gavage administration with a single dosage of $10 \mathrm{mg} / \mathrm{kg}$ body weight. Blood samples were collected in heparinized tubes via caudal vein predose and at 1,2, 3, 4, 5, 6, 8, 10 and $24 \mathrm{~h}$ postdose. Blood samples were immediately centrifuged at $6,000 \mathrm{rpm}$ for $10 \mathrm{~min}$ to obtain the plasma. Plasma samples were transferred to clean tubes and underwent the sample preparation procedures as described above before LC-MS/MS analysis. Pharmacokinetic parameters were calculated by non-compartmental analysis using the software of WinNonlin standard version 1.0 from Scientific Consulting Inc. (Apex, NC, USA).

\section{Results}

Growth inhibition of meisoindigo on the four human leukemic cells. As shown in Fig. 2, the leukemic cells grew well in the absence of meisoindigo with a relatively rapid proliferation rate, suggesting appropriate cell culture conditions were applied. The NB4, NB4.007/6 and HL60 cells proliferated 4-5 times during a 3-day period, while U937 proliferated about 7 times during the same period. There was always a delay in growth on the first day, before the growth was accelerated. In our preliminary study, the growth and/or proliferation of the test cells were observed to decline on the 4th day after culture, if no fresh medium was added. In order to avoid this fluctuation in growth due to change of media, the inhibitory effects of meisoindigo was studied over three days.

Meisoindigo effectively inhibited the growth and proliferation of the retinoic acid sensitive cells (NB4 and HL60), as well as the retinoic acid resistant cells (NB4.007/6 and U937). The inhibitory effects of meisoindigo on the growth and proliferation of various leukemic cells exhibited a dose-dependent characteristic. The $50 \%$ inhibitory concentrations $\left(\mathrm{IC}_{50}\right)$ were estimated based on the values of growth obtained after $72 \mathrm{~h}$ incubation and the data fitted to an Inhibitory Effect Sigmoid $\mathrm{E}_{\max }$ Model. The correlation of regression of the model fittings was 0.986 , indicating an appropriate pharmacodynamic model was applied. The $\mathrm{IC}_{50}$ values were 7.9, 7.1, 7.1 and $7.5 \mu \mathrm{M}$ in NB4, NB4.007/6, HL60 and U937 cells, respectively. No statistically significant difference among these $\mathrm{IC}_{50}$ values was observed. After exposure to meisoindigo at $8 \mu \mathrm{M}$ or above for $72 \mathrm{~h}$, all leukemic cells were clearly inhibited and there was a greater than $50 \%$ decrease in the number of viable cells in the meisoindigo treated group over the control (Fig. 2). When treated with $20 \mu \mathrm{M}$ meisoindigo for 48 to $72 \mathrm{~h}$, most of the cells did not survive. The antileukemic effects of meisoindigo seemed to be time-dependent. Prolonged incubation with high concentrations of meisoindgo $(12,16$ or $20 \mu \mathrm{M})$ led to enhanced inhibitory effects. The calculation of $\mathrm{IC}_{50}$ with counts on day 1,2 or 3 resulted in very similar finding in the four leukemic cells. Interestingly, the finding that meisoindigo was effective in inhibiting the growth and proliferation of retinoic acid resistant NB4.007/6 and U937 cells, suggests a less possibility of 

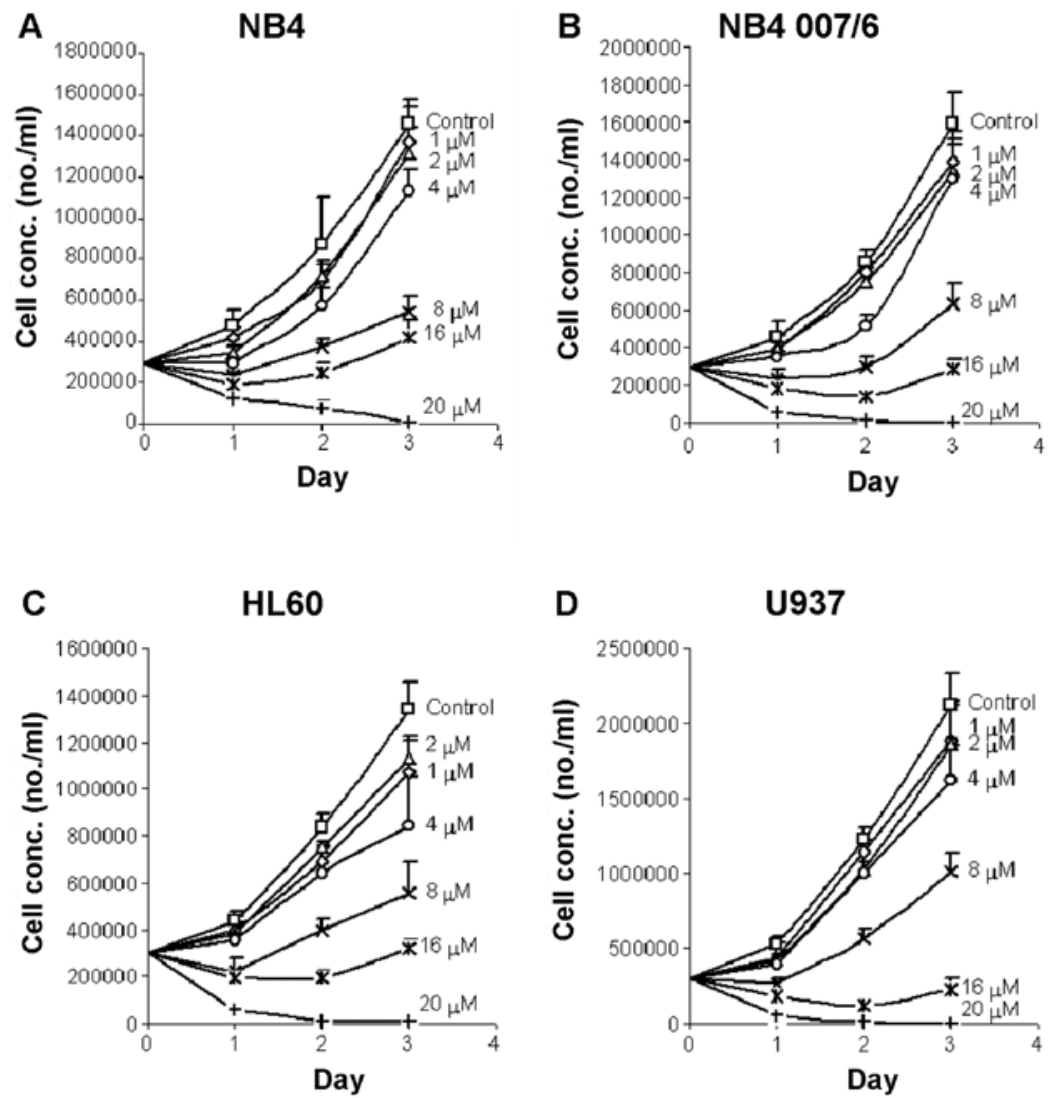

Figure 2. Effect of meisoindigo on the growth of four human leukemic cell types (A) NB4, (B) NB4.007/6, (C) HL-60 and (D) U937.

cross-resistance with retinoic acid. Thus meisoindigo could be a potential treatment for leukemia, either sensitive or resistant to retinoic acid.

Apoptosis induction of meisoindigo in four human leukemic cell types. The flow cytometry analysis showed that NB4, NB4.007/6, HL60 and U937 cells grew well in the absence of meisoindigo with about $20-30 \%$ of the cells distributed in the respective $\mathrm{M} / \mathrm{G} 2$ phase and $\mathrm{S}$ phase (Table II), suggesting that these cells have active DNA synthesis and cell division. This phenomenon was in good accord with the rapid growth and proliferation of these cells. After treatment with meisoindigo, the percentage of sub-G1 cells increased substantially over time in all the four cell types (Table II). The percentage of sub-G1 cells, which is regarded as the percentage of apoptosis shown in Fig. 3, indicated that the induction of apoptosis was both time- and dose-dependent. The percentages of sub-G1 cells in all the four cell types were quite similar at high concentrations of meisoindigo $(12,16,20 \mu \mathrm{M})$, suggesting that the apoptosis-inductive effect of meisoindigo was saturable at high concentrations. Also, the percentage of G1/G0, S and $\mathrm{M} / \mathrm{G} 2$ dropped substantially as the dosages increased (Table II), which suggested that no phase arrest or cell phase accumulation occurred in these meisoindigo-treated cells, and thus the antileukemic effects of meisoindigo in the cells might be independent of cell cycle arrest. However, the apoptotic effect is not associated with DNA fragmentation (data not shown).

LC-MS/MS method development. The LC conditions were optimized by varying the mobile phase, gradient elution,
Table II. Percentages in phase distribution of the leukemic cells after incubation with meisoindigo for $48 \mathrm{~h}$.

\begin{tabular}{lrrrr}
\hline Conc. & Sub-G1 & G1/G0 & S & M/G2 \\
\hline NB4 cells & & & & \\
0 & $6.2 \pm 0.9^{\mathrm{a}}$ & $46.6 \pm 0.7$ & $29.8 \pm 0.5$ & $17.8 \pm 1.0$ \\
8 & $36.8 \pm 2.2$ & $34.3 \pm 1.4$ & $19.8 \pm 0.1$ & $9.4 \pm 0.7$ \\
12 & $50.8 \pm 1.8$ & $30.1 \pm 0.7$ & $12.7 \pm 0.5$ & $6.4 \pm 0.6$ \\
NB4.007/6 cells & & & & \\
0 & $8.9 \pm 1.0$ & $38.2 \pm 1.0$ & $29.8 \pm 0.5$ & $23.4 \pm 1.5$ \\
8 & $17.6 \pm 2.4$ & $36.6 \pm 2.4$ & $27.9 \pm 0.1$ & $18.2 \pm 1.9$ \\
12 & $34.3 \pm 2.2$ & $29.1 \pm 2.2$ & $24.3 \pm 2.0$ & $12.9 \pm 1.6$ \\
HL60 cells & & & & \\
0 & $8.5 \pm 0.6$ & $45.4 \pm 2.1$ & $25.3 \pm 0.6$ & $21.1 \pm 1.5$ \\
8 & $48.8 \pm 0.8$ & $29.0 \pm 0.6$ & $15.0 \pm 0.4$ & $7.7 \pm 0.4$ \\
12 & $54.6 \pm 0.2$ & $28.9 \pm 0.3$ & $11.2 \pm 0.3$ & $5.8 \pm 0.3$ \\
U937 cells & & & & \\
0 & $1.1 \pm 0.3$ & $48.1 \pm 0.5$ & $24.5 \pm 1.3$ & $26.6 \pm 1.0$ \\
8 & $25.7 \pm 7.2$ & $37.5 \pm 3.0$ & $19.0 \pm 2.0$ & $18.1 \pm 2.5$ \\
12 & $39.9 \pm 5.1$ & $34.9 \pm 1.9$ & $15.0 \pm 1.0$ & $10.6 \pm 2.1$ \\
\hline
\end{tabular}

${ }^{a}$ Data was presented as mean \pm SD. $n=3$.

flow rate and columns. The MS conditions were optimized by tuning with authentic standards of meisoindigo, its reductive metabolites and indirubin. The proposed fragmentation scheme and MS/MS spectrum of protonated meisoindigo 

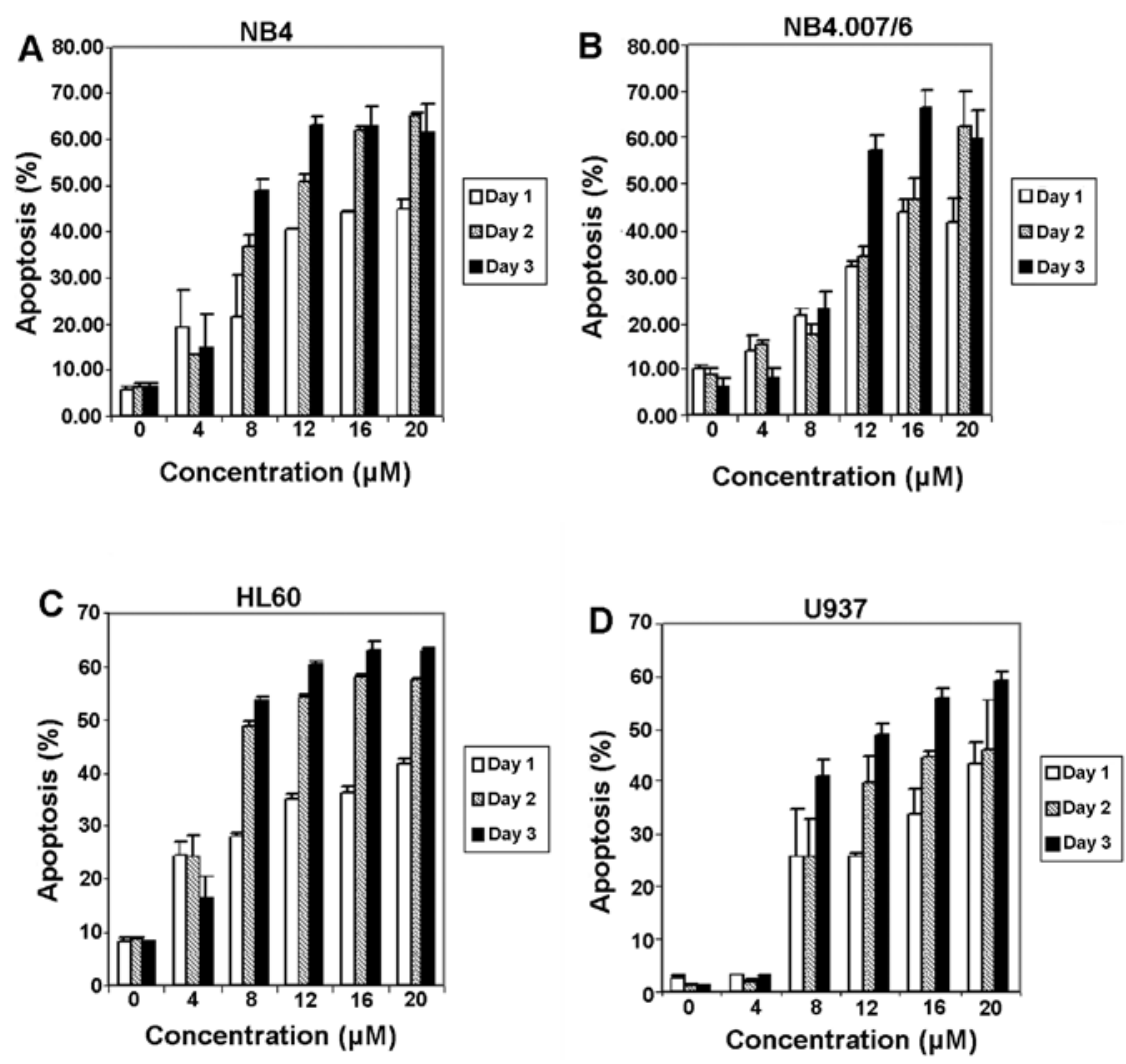

Figure 3. Dose- and time-dependent apoptosis induction effect of meisoindigo in four human leukemic cells (A) NB4, (B) NB4.007/6, (C) HL-60 and (D) U937.

$(m / z, 277)$ are shown in Fig. 4A. Loss of CONH (43 Da) following gain of one $\mathrm{H}$ generated a major product ion at $m / z$ 234. Loss of $\mathrm{CONCH}_{3}(57 \mathrm{Da})$ following loss of one $\mathrm{H}$ generated the most abundant product ion at $m / z 218$. The product ion at $m / z, 234$ was selected to form the MRM transition for quantification due to its better selectivity without compromising the sensitivity. As is shown in Fig. 1, the reductive metabolites of meisoindigo $\mathrm{M} 1+\mathrm{M} 2$ were a pair of (3-R, 3'-R) and (3-S, 3'-S) reduced-meisoindigo enantiomers with two hydrogens located at the same side of 3,3'-single bond, whereas M3+M4 were another pair of (3-R, 3'-S) and (3-S, 3'-R) enantiomers with two hydrogens located at the opposite sides. The MS/MS spectra of M1+M2 and M3+M4 were identical and are shown in Fig. 4B. The proposed fragmentation scheme of protonated reductive metabolites $(\mathrm{m} / \mathrm{z}, 279)$ showed that cleavage of the $3,3^{\prime}$ bond generated the dominant product ion at $m / z 147$, which was thus selected to form the MRM transition for quantification. The proposed fragmentation scheme and MS/MS spectrum of protonated indirubin $(m / z, 263)$ are shown in Fig. 4C. Loss of $\mathrm{CONH}$ (43 Da) and CO (28 Da) from indirubin following loss of one $\mathrm{H}$ generated the most abundant product ion at $\mathrm{m} / \mathrm{z} 190$. Thus the product ion at $\mathrm{m} / \mathrm{z} 190$ was selected to form the MRM transition for quantification.

LC-MS/MS method validation. Full validation was conducted for the pharmacokinetic study of meisoindigo and its reductive metabolites in rat plasma because this bioanalytical method was developed for the first time. The assay selectivity was confirmed as no significant interference was observed at the retention time of meisoindigo in the blank rat plasma. The matrix effect on the analyte determination was not taken into account due to the relative slow chromatographic separation. The linear range of calibration curve was $1-500 \mathrm{ng} / \mathrm{ml}$ with correlation coefficients greater than 0.9998. LLOQ was $1 \mathrm{ng} / \mathrm{ml}$ for meisoindigo (Fig. 5A). The linear range of calibration curve was $2.5-1,000 \mathrm{ng} / \mathrm{ml}$ with correlation coefficients greater than 0.9928 . LLOQ was $0.5 \mathrm{ng} / \mathrm{ml}$ for the reductive metabolites of meisoindigo (Fig. 5B).

Table III shows both intra- and inter-day accuracy and precision data determined by the analysis of meisoindigo and its reductive metabolites. These results fulfilled the criteria of validation with accuracy and precision not more than $\pm 20 \%$, indicating that this assay is consistent and reliable with good accuracy and precision. The extraction recoveries of low, medium, high concentrations were within $\pm 20 \%$ and reproducible. The results of short-term, long-term, freeze and thaw, stock solution and post-preparative stability evaluated at a low concentration of $5 \mathrm{ng} / \mathrm{ml}$ and a high concentration of $500 \mathrm{ng} / \mathrm{ml}$ of meisoindigo and its reductive metabolites in rat plasma were summarized in Table IV.

Pharmacokinetic profiles of meisoindigo and its reductive metabolites. The pharmacokinetic profiles of meisoindigo and its reductive metabolites in rat plasma are shown in Fig. 6. Data points were the average of four measurements with standard deviation (SD) as the error bars. The major pharmacokinetic parameters are listed in Table V. It is shown in Fig. 6 that meisoindigo was converted to its reductive metabolites rapidly, indicating the 3,3' double bond within the molecule of meisoin- 

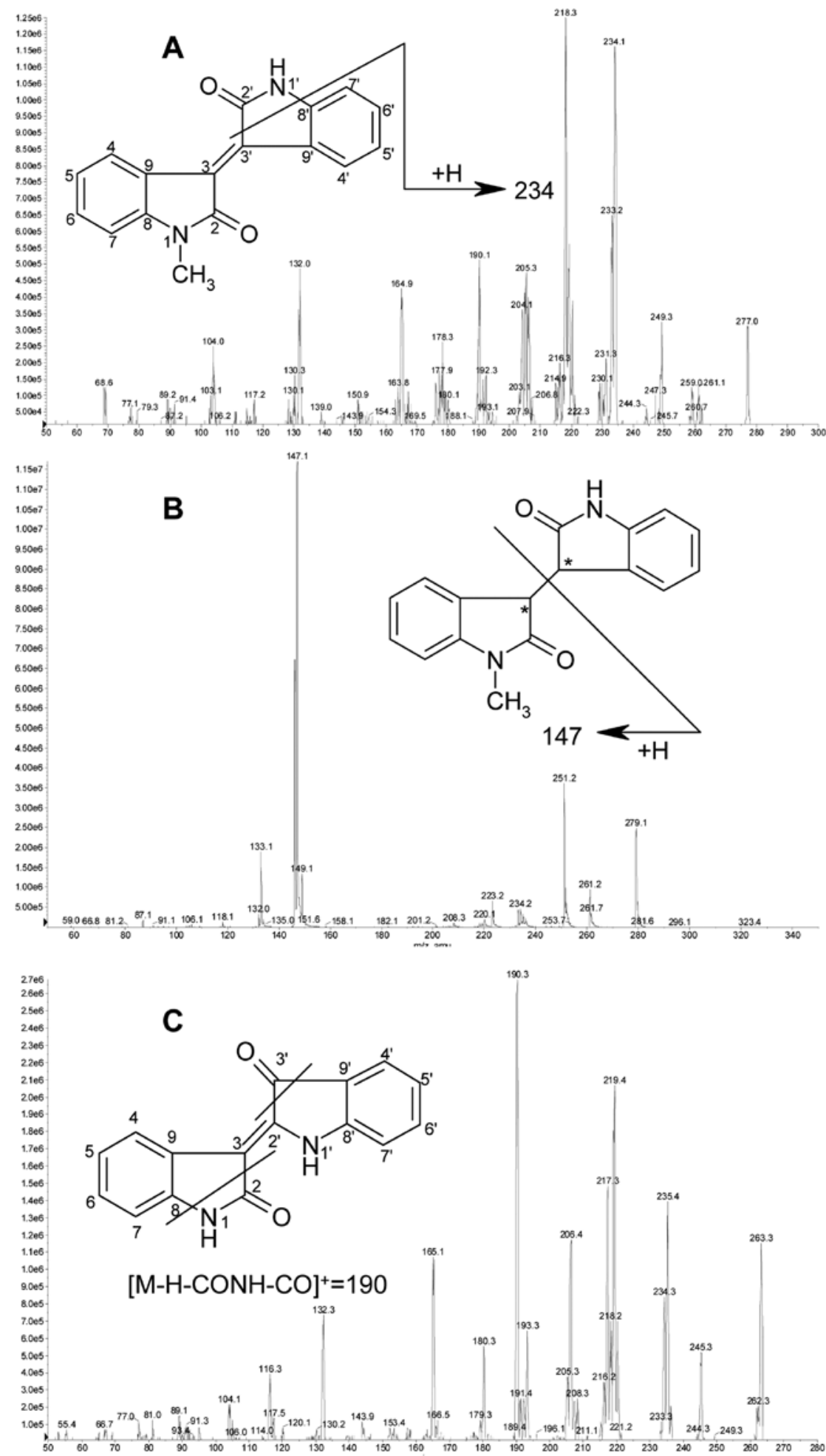

Figure 4. MS/MS spectra of protonated meisoindigo at $m / z 277$ (A), its reductive metabolites at $m / z 279$ (B), indirubin (internal standard) at $m / z, 263$ (C), and the proposed origin of key product ions.

digo is metabolically unstable. Plasma level of the parent drug was low, whereas plasma level of its reductive metabolites was much higher suggesting the occurrence of extensive first pass effect. The wide variation in plasma levels of the drug and its reductive metabolites also indicated possible incomplete absorption due to the poor biopharmaceutical properties of the drug. In Table $\mathrm{V}$, the low $\mathrm{AUC}_{0 \rightarrow 24 \mathrm{~h}}$ and long $t_{1 / 2}$ (a composite parameter determined by volume of distribution and clear- ance) could be partly caused by a large volume of distribution of meisoindigo due to its lipophilicity.

\section{Discussion}

It has been reported in previous in vitro studies that meisoindigo could effectively inhibit the growth and/or proliferation and induce apoptosis in the leukemic cells isolated from 

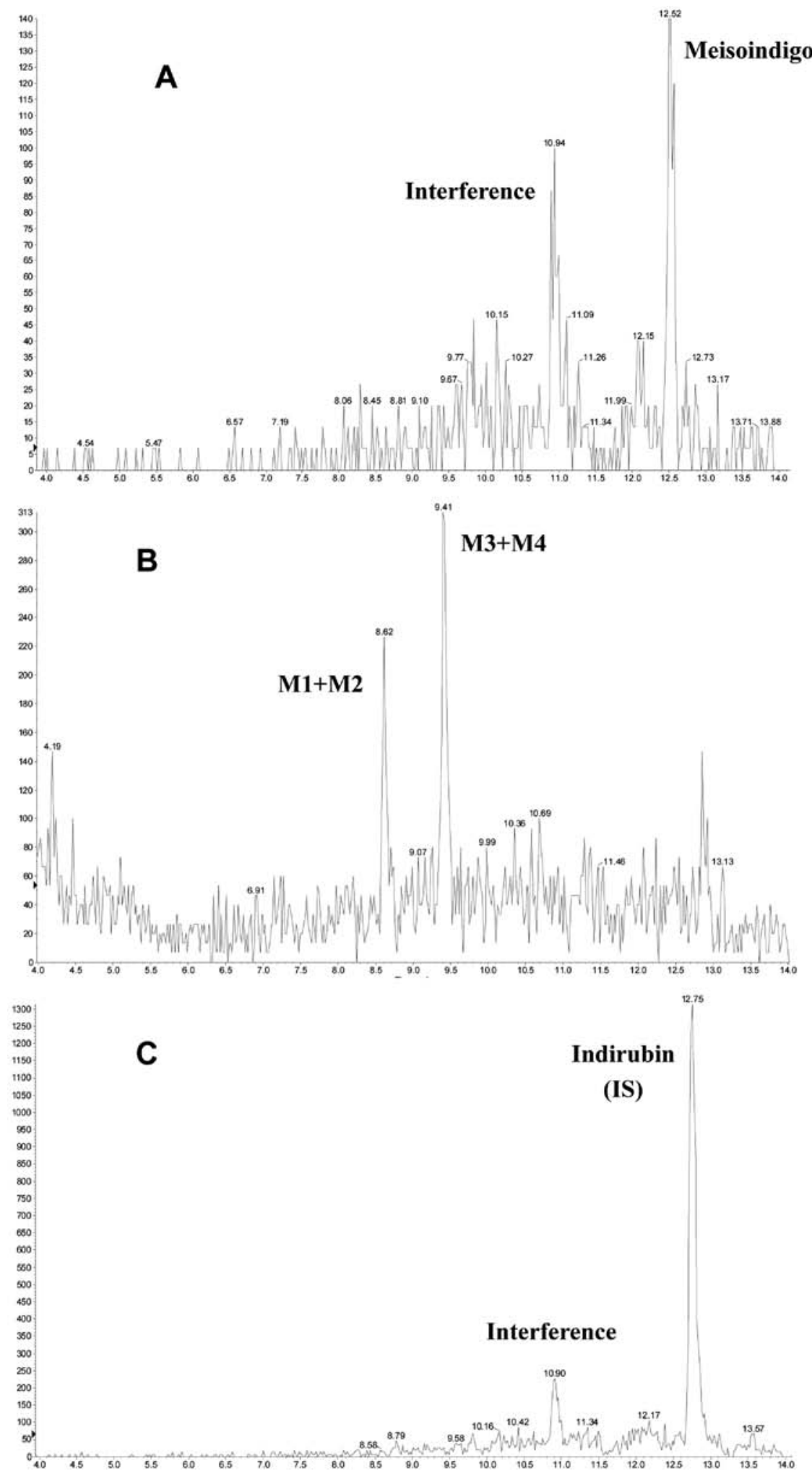

Figure 5. MRM chromatograms for (A) meisoindigo at lower limit of quantitation (1.0 ng/ml), (B) its reductive metabolites at lower limit of quantitation (0.5 ng/ml), and (C) indirubin (internal standard, $100 \mathrm{ng} / \mathrm{ml}$ ) in rat plasma.

newly diagnosed CML patients and CML K562 cells $(1,16)$. In the present study, a similar effect of meisoindigo was observed in the other four human leukemic cells, including NB4, NB4.007/6, HL60 and U937. $\mathrm{IC}_{50}$ of meisoindigo for growth inhibition of the four human leukemic cell types ranged from 7-8 $\mu \mathrm{M}$; and at concentration of $12 \mu \mathrm{M}$, meisoindigo was able to effectively induce apoptosis in all the cells. These concentrations were even lower than the effective concentration of meisoindigo $(20 \mu \mathrm{M})$ in K562 cells, a cell line derived from a CML patient in blast crisis (16). As the clinical efficacy of meisoindigo for CML has been well documented $(1,2)$, similar efficacy would be expected in the clinical treatment of other leukemias, such as APL and AML. 
Table III. Intra- and inter-day accuracy and precision $(n=3)$ for meisoindigo and its reductive metabolites in male rat plasma.

\begin{tabular}{|c|c|c|c|c|c|c|c|}
\hline \multicolumn{8}{|c|}{ Meisoindigo } \\
\hline Spiked concentration $(\mathrm{ng} / \mathrm{ml})$ & 1 & 2.5 & 5 & 10 & 50 & 250 & 500 \\
\hline Intra-day accuracy (\%) & 113.8 & 92.3 & 101.5 & 103.2 & 104.1 & 97.9 & 97.0 \\
\hline Intra-day RSD (\%) & 4.1 & 10.6 & 1.7 & 18.0 & 11.7 & 11.6 & 2.3 \\
\hline Inter-day accuracy (\%) & 97.1 & 95.4 & 106.3 & 88.9 & 95.6 & 100.2 & 102.4 \\
\hline Inter-day RSD (\%) & 15.6 & 17.3 & 2.9 & 18.8 & 11.3 & 10.4 & 7.3 \\
\hline \multicolumn{8}{|c|}{ Reductive metabolites } \\
\hline Spiked concentration (ng/ml) & 2.5 & 5 & 50 & 100 & 250 & 500 & 1,000 \\
\hline Intra-day accuracy (\%) & 97.9 & 110.6 & 85.2 & 100.5 & 97.3 & 107.3 & 102.5 \\
\hline Intra-day RSD (\%) & 5.4 & 10.8 & 3.3 & 1.9 & 6.6 & 6.9 & 5.3 \\
\hline Inter-day accuracy (\%) & 107.6 & 110.6 & 86.0 & 95.5 & 101.8 & 87.0 & 106.6 \\
\hline Inter-day RSD (\%) & 5.0 & 10.5 & 8.5 & 4.9 & 9.3 & 14.9 & 8.8 \\
\hline
\end{tabular}

Table IV. Stability of meisoindigo and its reductive metabolites in male rat plasma under various conditions.

\begin{tabular}{lcccc}
\hline & \multicolumn{3}{c}{ Percentage of initial value (mean $\pm \mathrm{SD} \%, \mathrm{n}=3)$} \\
Reductive metabolites
\end{tabular}

Table V. Pharmacokinetics parameters (mean $\pm \mathrm{SD}, \mathrm{n}=4$ ) of meisoindigo and its reductive metabolites in rat plasma after oral administration of dosage $10 \mathrm{mg} / \mathrm{kg}$.

\begin{tabular}{lcc}
\hline Parameters & Meisoindigo & Reductive metabolites \\
\hline $\mathrm{AUC}_{0 \rightarrow 24 \mathrm{~h}}(\mathrm{ng} \cdot \mathrm{h} / \mathrm{ml})$ & $19.6 \pm 15.8$ & $148.3 \pm 33.1$ \\
$T_{\max }(\mathrm{h})$ & $2.5 \pm 2.1$ & $1.4 \pm 0.2$ \\
$t_{1 / 2}(\mathrm{~h})$ & - & $3.6 \pm 1.5$ \\
$C_{\max }(\mathrm{ng} / \mathrm{ml})$ & $1.9 \pm 0.1$ & $38.8 \pm 10.7$ \\
\hline
\end{tabular}

It is interesting to note that meisoindigo is active in both the retinoic acid-sensitive APL cells (NB4) and retinoic acidresistant APL cells (NB4.007/6). This could be explained by the different antileukemic mechanisms of retinoic acid and meisoindigo. Retinoic acid exerts its antileukemic effects mainly through differentiation induction (17), while meisoindigo probably exerts its effects through induction of apoptosis and/ or differentiation, depending on the concentration. It might be beneficial to apply meisoindigo in the management of retinoic acid resistant APL. However, such postulation needs to be established through further in vitro and in vivo pre-clinical studies.

In this study, flow cytometry and cytomorphology results showed that the therapeutic effects of meisoindigo in the four

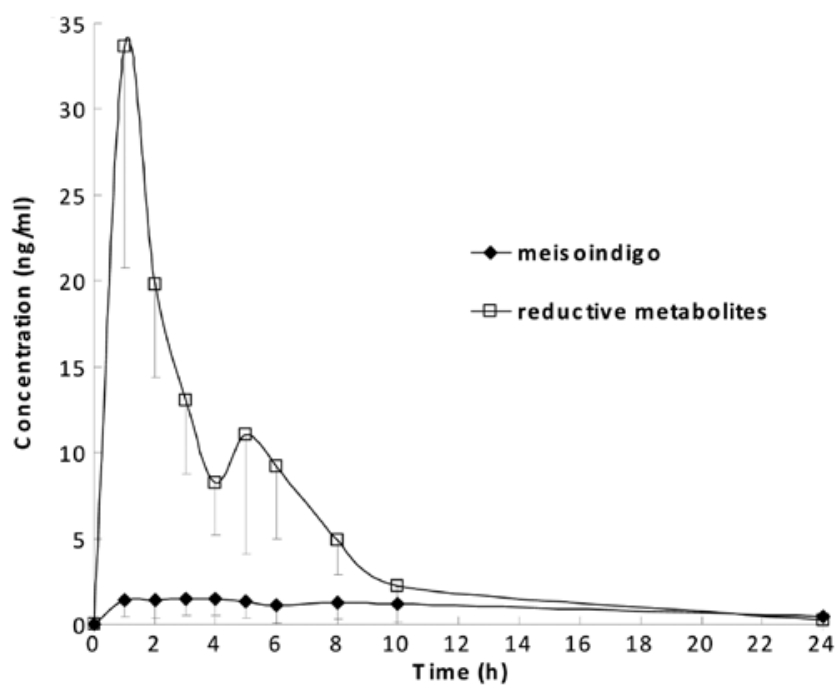

Figure 6. Concentration - time profiles of meisoindigo and its reductive metabolites in plasma obtained from four rats after oral administration.

human leukemic cells were related to apoptosis, while the apoptotic process was not associated with DNA fragmentation. Though the detection of the DNA fragments by gel electrophoresis, as a DNA ladder, is currently used as the major biochemical index of apoptosis, apoptosis can also 
occur without such phenomenon $(18,19)$. It is well known that internucleosomal cleavage of DNA is a later event in the apoptotic process. Key morphological changes of apoptosis can be dissociated experimentally from the DNA fragmentation produced by endonuclease activity if the apoptosis is induced in the presence of an inhibitor of endonuclease $(18,19)$. In light of the findings from the previous study (16) and the present study, we postulate that meisoindigo might be an inhibitor of endonuclease or that it induced apoptosis in these leukemic cells through an endonuclease-independent process. The molecular mechanisms for the apoptosis induction effects of meisoindigo are still unknown. Several possible mechanisms might be involved, including downregulation of $b c l-2$ gene and/or pathogenic oncogene PML-RAR $\alpha$; phase arrest through inhibition of CDKs; inhibition of DNA synthesis; inhibition of assembly of microtubule protein; and partial induction of differentiation $(1-4,16,20)$. The antileukemic effects of meisoindigo could be the combined effects of the above-mentioned functions.

As the clinical usage of meisoindigo is mainly through oral administration, pharmacokinetic parameters were determined from plasma in rats following oral administration of meisoindigo in this study. The low and variable level of the parent drug meisoindigo in rat plasma shown from in vivo pharmacokinetics studies could be due to extensive first pass effect and absorption incompleteness and variation. The latter problem could partially be rectified by formulation modification. It was found in our previous study that, compared with the metabolic profile of meisoindigo in rat liver microsomes, the types of metabolites produced in rat small intestine microsomes are much less and their quantities are much lower (21). Therefore, it could be deduced that the in vivo extensive first pass effect of meisoindigo is mainly due to its metabolism in the liver.

Interestingly, an apparent disconnection between in vitro and in vivo pharmacological data of meisoindigo was revealed in this study. It was reported that the production of vascular endothelial growth factor (VEGF) secreted by human CML cells was decreased, and time-dependent apoptosis of human ECV304 cells was induced, after treatment with $10 \mu \mathrm{M}$ meisoindigo (5). This required concentration for the respective effects is much higher than the in vivo nano-level plasma concentration of meisoindigo observed in this study (slightly above $1 \mathrm{ng} / \mathrm{ml}$ ). This in vivo plasma concentration in rats could reflect the plasma concentration of meisoindigo in humans, considering no significant difference in the metabolic stability profiles of meisoindigo between rat and human (12) as well as the comparable dosages used in our rat model $(10 \mathrm{mg} / \mathrm{kg})$ and in human application (100-150 mg/day) (2). However, it has been reported that meisoindigo was equally efficient for both newly diagnosed and previously treated CML patients (2). Therefore, the contradicting finding of the observed sub-therapeutic plasma concentration of the parent drug and clinical efficacy could be a strong indication of the presence of active metabolites of meisoindigo, which led to a greater in vivo pharmacological response inconsistent with in vitro biological data and in vivo pharmacokinetics profile (22).

Since the reductive metabolites of meisoindigo are dominant in rat plasma (13), they were synthesized as authentic standards and thus quantified in this study. The reductive metabolites of meisoindigo are actually comprised of two pairs of enantiomers (M1+M2, M3+M4), which can be eluted as two peaks on conventional HPLC column and four peaks on Chiral HPLC column (11). Unfortunately, it was observed that one pair of enantiomers is unstable in the liquid state and converts gradually into the other pair of enantiomers (11). Thus, it is challenging to accurately quantify the two peaks on conventional HPLC column or four peaks on Chiral HPLC column. In this study, conventional HPLC column was chosen and the peak area sum of the two peaks corresponding to two pairs of enantiomers was used to quantify the total amounts of reductive metabolites of meisoindigo in rat plasma. A previous study suggested that an intact exocyclic double bond was essential to maintain planarity and rigidity of the isoindigo scaffold, a feature essential for its activity (23). Since the reductive metabolites of meisoindigo lost the double bond in their structures, they are anticipated to be inactive.

In our previous studies, the in vivo metabolic profiles of meisoindigo in rat urine and feces were also examined qualitatively (13). The parent drug and its reductive metabolites existed in rat urine with trace amount which is close to LOD and thus was not analyzed quantitatively. It is unnecessary to quantify the parent drug and its reductive metabolites in rat feces, because both of them are absent in feces samples. Prospective studies involving the quantitative analysis of the dominant metabolites of meisoindigo at $\mathrm{m} / \mathrm{z} 295$ undergone reduction followed by phenyl mono-oxidation as a function of time in rat urine and feces could be considered to provide a more comprehensive in vivo metabolic profile of this drug.

In conclusion, the antileukemic effects of meisoindigo were investigated in four human leukemic cells (NB4, NB4.007/6, HL-60 and U937) including both retinoic acid sensitive and retinoic acid resistant cells. We found that meisoindigo could effectively inhibit the growth and/or proliferation of these four cell types at $\mu \mathrm{M}$ levels. The effects of meisoindigo in these cells are related to its proliferation inhibition and apoptosis induction, and are independent of cell cycle arrest. However, the apoptotic effect is not associated with DNA fragmentation. Clinical efficacy of meisoindigo could be possible in the treatment of other leukemias, such as APL and AML. It might be beneficial to apply meisoindigo in the management of retinoic acid-resistant APL. In addition, a sensitive and selective LC-MS/MS method was developed and validated for simultaneous determination of meisoindigo and its reductive metabolites whose concentration are at different order of magnitude in rat plasma. The profiles of plasma concentration versus time were plotted and the relevant pharmacokinetic parameters were calculated for meisoindigo and its reductive metabolites. Poor pharmacokinetic characteristics of meisoindigo could be partly caused by the poor biopharmaceutical properties of the drug. It warrants exploring whether formulation modification could improve some of these pharmacokinetic characteristics, such as the bioavailability of this drug. In particular, the contradiction of poor pharmacokinetic characteristics and clinical efficacy could be a strong indication of the presence of active metabolites of meisoindigo.

\section{Acknowledgements}

This study was financially supported by the National University of Singapore Academic Research Fund R148-000-104-112. 


\section{References}

1. Xiao Z, Qian L, Liu B and Hao Y: Meisoindigo for the treatment of chronic myelogenous leukaemia. Br J Haematol 111: 711-712, 2000

2. Xiao Z, Hao Y, Liu B and Qian L: Indirubin and meisoindigo in the treatment of chronic myelogenous leukemia in China. Leuk Lymphoma 43: 1763-1768, 2002.

3. Ji XJ, Liu XM, Li K, Chen RH and Wang LG: Pharmacological studies of meisoindigo: absorption and mechanism of action. Biomed Environ Sci 4: 332-337, 1991.

4. Liu XM, Wang LG, Li HY and Ji XJ: Induction of differentiation and down-regulation of c-myb gene expression in ML-1 human myeloblastic leukemia cells by the clinically effective anti-leukemia agent meisoindigo. Biochem Pharmacol 51: $1545-1551,1996$

5. Xiao Z, Wang Y, Lu L, et al: Anti-angiogenesis effects of meisoindigo on chronic myelogenous leukemia in vitro. Leuk Res 30: 54-59, 2006.

6. Lee CC, Lin CP, Lee YL, Wang GC, Cheng YC and Liu HE: Meisoindigo is a promising agent with in vitro and in vivo activity against human acute myeloid leukemia. Leuk Lymphoma 51: 897-905, 2010.

7. Lanotte M, Martin-Thouvenin V, Najman S, Balerini P, Valensi F and Berger R: NB4, a maturation inducible cell line with $\mathrm{t}(15 ; 17)$ marker isolated from a human acute promyelocytic leukemia (M3). Blood 77: 1080-1086, 1991.

8. Breitman TR, Selonick SE and Collins SJ: Induction of differentiation of the human promyelocytic leukemia cell line (HL-60) by retinoic acid. Proc Natl Acad Sci USA 77: 2936-2940, 1980.

9. Dermime S, Grignani F, Rogaia D, Liberatore C, Marchesi E and Gambacorti-Passerini C: Acute promyelocytic leukaemia cells resistant to retinoic acid show further perturbation of the RAR alpha signal transduction system. Leuk Lymphoma 16: 289-295, 1995 .

10. Olsson IL and Breitman TR: Induction of differentiation of the human histiocytic lymphoma cell line U-937 by retinoic acid and cyclic adenosine $3^{\prime}: 5^{\prime}$-monophosphate-inducing agents. Cancer Res 42: 3924-3927, 1982.

11. Huang M, Goh LT and Ho PC: Identification of stereoisomeric metabolites of meisoindigo in rat liver microsomes by achiral and chiral liquid chromatography/tandem mass spectrometry. Drug Metab Dispos 36: 2171-2184, 2008.
12. Huang M and Ho PC: Identification of metabolites of meisoindigo in rat, pig and human liver microsomes by UFLC-MS/MS. Biochem Pharmacol 77: 1418-1428, 2009.

13. Huang M, Lee YS and Ho PC: Identification of circulatory and excretory metabolites of meisoindigo in rat plasma, urine and feces by high-performance liquid chromatography coupled with positive electrospray ionization tandem mass spectrometry. Rapid Commun Mass Spectrom 24: 729-741, 2010.

14. Collins SJ, Gallo RC and Gallagher RE: Continuous growth and differentiation of human myeloid leukaemic cells in suspension culture. Nature 270: 347-349, 1977.

15. Sundstrom $C$ and Nilsson K: Establishment and characterization of a human histiocytic lymphoma cell line (U-937). Int J Cancer 17: 565-577, 1976.

16. Song L and Qian L: Apoptosis inducing effect of meisoindigo on K562 cells. Zhongguo Zhong Xi Yi Jie He Za Zhi 19: 353-355, 1999 (In Chinese).

17. Lin RJ, Egan DA and Evans RM: Molecular genetics of acute promyelocytic leukemia. Trends Genet 15: 179-184, 1999.

18. Bedner E, Li X, Gorczyca W, Melamed MR and Darzynkiewicz Z: Analysis of apoptosis by laser scanning cytometry. Cytometry 35: 181-195, 1999.

19. Cohen GM, Sun XM, Snowden RT, Dinsdale D and Skilleter DN: Key morphological features of apoptosis may occur in the absence of internucleosomal DNA fragmentation. Biochem J 286: 331-334, 1992.

20. Hoessel R, Leclerc S, Endicott JA, et al: Indirubin, the active constituent of a Chinese antileukaemia medicine, inhibits cyclin-dependent kinases. Nat Cell Biol 1: 60-67, 1999.

21. Huang M, Choo LW and Ho PC: Characterization of metabolites of meisoindigo in male and female rat kidney microsomes by high-performance liquid chromatography coupled with positive electrospray ionization tandem mass spectrometry. Rapid Commun Mass Spectrom 22: 3835-3845, 2008.

22. Fura A: Role of pharmacologically active metabolites in drug discovery and development. Drug Discov Today 11: 133-142, 2006.

23. Wee XK, Yeo WK, Zhang B, et al: Synthesis and evaluation of functionalized isoindigos as antiproliferative agents. Bioorg Med Chem 17: 7562-7571, 2009. 